\title{
Figures of Merit for Control Verification
}

\author{
Luis G. Crespo* \\ National Institute of Aerospace \\ Sean P. Kenny and Daniel P. Giesy ${ }^{\dagger}$ \\ Dynamic Systems and Control Branch, NASA Langley Research Center
}

This paper proposes a methodology for evaluating a controller's ability to satisfy a set of closed-loop specifications when the plant has an arbitrary functional dependency on uncertain parameters. Control verification metrics applicable to deterministic and probabilistic uncertainty models are proposed. These metrics, which result from sizing the largest uncertainty set of a given class for which the specifications are satisfied, enable systematic assessment of competing control alternatives regardless of the methods used to derive them. A particularly attractive feature of the tools derived is that their efficiency and accuracy do not depend on the robustness of the controller. This is in sharp contrast to Monte Carlo based methods where the number of simulations required to accurately approximate the failure probability grows exponentially with its closeness to zero. This framework allows for the integration of complex, high-fidelity simulations of the integrated system and only requires standard optimization algorithms for its implementation.

Keywords: control verification, uncertainty, robustness, probability.

\section{Acronyms}

CPV : Critical Parameter Value

CSR : Critical Similitude Ratio

MS : Maximal Set

PSM : Parametric Safety Margin

RI : Reliability Index

\section{Introduction}

Most of the strategies for robust control design are based on attaining satisfactory worst-case stability and performance [1-5]. While $H_{\infty}$ and $\mu$-analysis and synthesis ${ }^{3}$ are the best methods for dealing with unstructured uncertainty, their intrinsic formulation render them overly conservative when dealing with parametric uncertainty. These methods usually lead to high-order controllers due to this conservatism and their emphasis on the worst-case. On the other hand, the control literature on parametric uncertainty has been mostly devoted to the robust stability of linear time invariant systems having affine and multi-affine parameter ${ }^{6-9}$ dependencies. Notable exceptions to this case are made in References $[10,11]$ where polynomial parameter dependencies and approximate feasibility are considered. However, the deployment of these strategies over other functional

${ }^{*}$ Senior Staff Scientist, 100 Exploration Way, Hampton VA 23666 USA. AIAA Professional Member.

${ }^{\dagger}$ Aerospace Technologists, Mail Stop 308, NASA LaRC, Hampton VA 23681 USA. 
forms requires over-bounding, a practice that introduces conservatism into the robustness analysis. Realistic control applications are subject to both stability and performance requirements that have an arbitrary functional dependency on the uncertain parameters and where the effect of the non-linear dynamics is important.

The verification of satisfactory control effectiveness is a crucial task for safety-critical applications where instability and poor performance may lead to the loss of a mission. Since this is the last software-based step in the control design cycle before control validation, the best dynamic model must be used. This leads to complex, non-linear, high-fidelity simulations where all subsystems, commonly developed independently with each using its own set of assumptions and methods, are integrated. Under these conditions, the vast majority of assumptions behind robust and adaptive control methods - e.g., linear dynamics, affine or multi-affine parameter dependencies, attainment of matching conditions - do not hold. Even though such assumptions enable the efficient manipulation of the problem and the generation of computationally viable control solutions, the physics of the system, or, at this stage, our best model of it, will ultimately validate the control practices exercised thus far. In accordance with this rationale, the framework proposed herein will develop mathematics applicable to any representation of the dynamic system, including the best one available. In the context of robustness analysis, this implies that the structure of the plant's model is arbitrary and the controller, possibly designed using a simpler dynamic model based on a set of assumptions which we do not need to know, is available.

The nature and scope of this more general problem commonly leads to the usage of samplingbased techniques, e.g., Monte Carlo analysis. When accurate robustness assessments are desired, the computational demands associated with these methods render them impractical. This is especially true when the number of uncertain parameters is large and when probabilities are small. To alleviate this limitation, this paper proposes an optimization-based methodology and a set of metrics for control verification applicable to systems subject to both robust stability and robust performance requirements. The framework derived is applicable to linear and nonlinear systems having an arbitrary control structure. Besides, the functional relationship between the design requirements and the uncertainty may only be known implicitly, e.g., the dependence of the time response of a nonlinear system on the uncertain parameters. Robustness will be quantified using both deterministic and probabilistic uncertainty models. The figures of merit for evaluating robustness are the Parametric Safety Margin, the Reliability Index, the Failure Probability, and upper bounds to this probability. The tools for the calculation of these metrics, which are applications to control of methods developed by the authors ${ }^{12,13}$ only require the use of standard optimization algorithms.

This paper is organized as follows. Basic concepts and notions are first introduced in Section II. Section III presents the mathematical background required to perform set deformations, an instrumental tool for the developments that follow. The formulation and tools required to calculate the PSM are presented in Section IV, and the tools corresponding to the probabilistic metrics are presented in Section V. The strategies proposed are exercised in the study of eleven controllers designed for the robust control challenge problem posed at the 1990 American Control Conference. ${ }^{26}$ Finally, a few concluding remarks close the paper. Extensions of this framework to control analysis and tuning are made in the companion paper [14].

\section{Concepts and Notions}

The object of this paper is the evaluation of the robustness characteristics of closed-loop systems having a parametric mathematical model. The parameters which specify the closed-loop system are grouped into two categories: uncertain parameters, which are denoted by the vector $\boldsymbol{p}$, and the control design parameters, which are denoted by the vector $\boldsymbol{d}$. While the plant model depends on $\boldsymbol{p}$, the controller depends on $\boldsymbol{d}$. 
The uncertainty model of $\boldsymbol{p}$ can be deterministic or probabilistic. A deterministic uncertainty model is prescribed by the Uncertainty Set $\Delta$, while a probabilistic one is prescribed by a random vector. This vector is specified by the joint probability density function $f_{\boldsymbol{p}}(\boldsymbol{p})$ defined over $\Delta$. The uncertainty set of the probabilistic model is commonly called the Support Set. Hereafter, the terms uncertainty set and support set will be used interchangeably. By specifying the uncertainty model, it is implied that $\Delta$ contains the actual value of $\boldsymbol{p}$, which is assumed to be time invariant. In principle, the uncertainty set can be unbounded and can even be the entire parameter space. In practical applications, the choice of this model is usually made by a discipline expert. However, the theory presented herein acknowledges that such a choice may be fairly arbitrary, and addresses the need for quantifying the level of tolerance of the controller to uncertainty in $\boldsymbol{p}$ as the closed-loop performance degrades. Any member of the uncertainty set is called a Realization. The Nominal Parameter value, denoted as $\overline{\boldsymbol{p}}$, is a parameter realization regarded as a good deterministic representation of $\boldsymbol{p}$. Further, we will call the set of control design parameters of a baseline controller the Nominal Design point, $\overline{\boldsymbol{d}}$.

Stability and performance requirements for the closed-loop system will be prescribed by the set of constraint functions, $\boldsymbol{g}(\boldsymbol{p}, \boldsymbol{d}) \leq \mathbf{0}$, which depend on the uncertain parameters and the control parameters $^{\mathrm{a}}$. Throughout this paper, it is assumed that vector inequalities hold component wise. The larger the region in $\boldsymbol{p}$-space where $\boldsymbol{g}(\boldsymbol{p}, \overline{\boldsymbol{d}}) \leq \mathbf{0}$, the better the baseline controller. In the ideal case, such a region contains the support set $\Delta$. Note that this implies that an infinite number of constraints must be satisfied, specifically, $\operatorname{dim}(\boldsymbol{g})$ for each parameter realization.

Sets in the parameter and design spaces, instrumental to the developments that follow, are introduced next. The Failure Domain is given by ${ }^{\mathrm{b}}$

$$
\begin{gathered}
\mathcal{F}_{\boldsymbol{p}, \boldsymbol{d}}^{j} \triangleq\left\{\langle\boldsymbol{p}, \boldsymbol{d}\rangle: \boldsymbol{g}_{j}(\boldsymbol{p}, \boldsymbol{d}) \geq 0\right\}, \\
\mathcal{F}_{\boldsymbol{p}, \boldsymbol{d}} \triangleq \bigcup_{j=1}^{\operatorname{dim}(\boldsymbol{g})} \mathcal{F}_{\boldsymbol{p}, \boldsymbol{d}}^{j}
\end{gathered}
$$

While Equation (1) describes the failure domain corresponding to the $j$ th requirement, Equation (2) describes the failure domain for all requirements. The Non-Failure Domain is the complement set of the failure domain and will be denoted" as $\mathcal{F}^{c}$. The names "failure domain" and "non-failure domain" are used because in the failure domain at least one constraint is violated while, in the non-failure domain, all constraints are satisfied. The solution set of equation $\max _{j}\left\{\boldsymbol{g}_{j}\right\}=0$ usually partitions the space into these two domains. For control analysis purposes, the design point $\boldsymbol{d}$ will be kept at its nominal value $\overline{\boldsymbol{d}}$. In such a case, the projection of the failure domain onto the parameter space is given by

$$
\begin{aligned}
& \mathcal{F}_{\boldsymbol{p}}^{j}(\overline{\boldsymbol{d}}) \triangleq\left\{\boldsymbol{p}:\langle\boldsymbol{p}, \overline{\boldsymbol{d}}\rangle \in \mathcal{F}_{\boldsymbol{p}, \boldsymbol{d}}^{j}\right\}, \\
& \mathcal{F}_{\boldsymbol{p}}(\overline{\boldsymbol{d}}) \triangleq\left\{\boldsymbol{p}:\langle\boldsymbol{p}, \overline{\boldsymbol{d}}\rangle \in \mathcal{F}_{\boldsymbol{p}, \boldsymbol{d}}\right\} .
\end{aligned}
$$

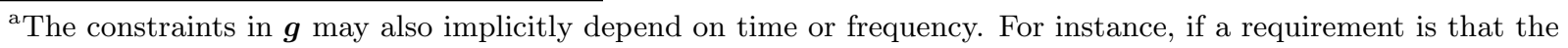
step response $y(\boldsymbol{p}, \boldsymbol{d}, t)$ must not exceed the upper envelope $\hat{y}(t)$, the corresponding constraint is given by

$$
\boldsymbol{g}=\max _{t}\{y(\boldsymbol{p}, \boldsymbol{d}, t)-\hat{y}(t)\} .
$$

Maximizations over $t$, such as this one, will be evaluated by selecting the largest value assumed by the argument at the discrete points of a time simulation. Hence, the evaluation of $\boldsymbol{g}$ will not entail solving an optimization problem per se.

${ }^{\mathrm{b}}$ Throughout this paper, super-indices are used to denote a particular vector or set while numerical sub-indices refer to vector components, e.g., $\boldsymbol{p}_{i}^{j}$ is the $i$ th component of the vector $\boldsymbol{p}^{j}$.

${ }^{\mathrm{c}}$ The complement set operator will be denoted as the super-index $c$. 
In the context of control analysis, which is the context of this paper, these two sets are also referred to as failure domains and are denoted as $\mathcal{F}^{j}$ and $\mathcal{F}$ for simplicity in the notation.

The Feasible Design Space, $\mathcal{E}$ and the Robust Design Space, $\mathcal{Q}$, are given by

$$
\begin{gathered}
\mathcal{E}(\overline{\boldsymbol{p}}) \triangleq\{\boldsymbol{d}: \boldsymbol{g}(\overline{\boldsymbol{p}}, \boldsymbol{d})<\mathbf{0}\}, \\
\mathcal{Q}(\Delta) \triangleq\{\boldsymbol{d}: \boldsymbol{g}(\boldsymbol{p}, \boldsymbol{d})<\mathbf{0}, \forall \boldsymbol{p} \in \Delta\} .
\end{gathered}
$$

The controller with gains $\overline{\boldsymbol{d}}$ will be called Robust if $\mathcal{F}$ and $\Delta$ do not overlap. In such a case, $\overline{\boldsymbol{d}}$ belongs into the robust design space. Otherwise, the controller will be called Non-Robust. The level of robustness of a controller is related to the size and geometry of the non-failure domain. Note that $\mathcal{Q} \subset \mathcal{E}$ when $\bar{p} \in \Delta$.

\section{Set Deformations}

Some of the figures of merit proposed will result from deforming sets in the parameter space $\boldsymbol{p}$. The mathematical background for such deformations is introduced next.

Let $\Omega$ be a set in $\boldsymbol{p}$-space, called the Reference Set, whose geometric center is the nominal parameter $\overline{\boldsymbol{p}}$. The geometry of $\Omega$ will be prescribed according to the relative levels of uncertainty in $\boldsymbol{p}$. One possible choice for the reference set is a hyper-sphere. The hyper-sphere of radius $R$ centered at $\overline{\boldsymbol{p}}$, denoted as $\mathcal{S}(\overline{\boldsymbol{p}}, R)$, is defined by

$$
\mathcal{S}(\overline{\boldsymbol{p}}, R)=\{\boldsymbol{p}:\|\overline{\boldsymbol{p}}-\boldsymbol{p}\| \leq R\}
$$

where $\|\cdot\|$ denotes the Euclidean norm. Another choice might be to confine each component of the reference set to a bounded interval. This leads to a hyper-rectangular set. If $\boldsymbol{m}>\mathbf{0}$ is the vector of half-lengths of such a set, the hyper-rectangle $\mathcal{R}(\overline{\boldsymbol{p}}, \boldsymbol{m})$ is defined by

$$
\mathcal{R}(\overline{\boldsymbol{p}}, \boldsymbol{m})=\{\boldsymbol{p}: \overline{\boldsymbol{p}}-\boldsymbol{m} \leq \boldsymbol{p} \leq \overline{\boldsymbol{p}}+\boldsymbol{m}]\} .
$$

Parameters with similar levels of uncertainty can be modeled using hyper-spherical sets. These sets could also be used for parameters with dissimilar levels of uncertainty if some scaling is used. However, whether scaling is used or not, a degree of dependence among parameters is introduced. For instance, the range of variability of one parameter depends on the values taken on by the others. Rectangular sets permit the consideration of dissimilar levels of uncertainty without the need for scaling and introduce no such dependence. Such levels are attained by making $\boldsymbol{m}_{i}$ proportional to the level of uncertainty in $\boldsymbol{p}_{i}$.

For the sake of clarity, the presentation that follows concentrates on the case where the nominal design point belongs to $\mathcal{E}$, i.e., when the controller satisfies the requirements for the nominal plant. One of the tasks of interest is to assign a measure of robustness to a controller based on measuring how much the reference set can be deformed before intersecting the failure domain. This requires specifying what we mean by a deformation. The Homothetic Deformation of $\Omega$ with respect to the

nominal parameter point $\overline{\boldsymbol{p}}$ by a factor of $\alpha \geq 0$, is the set $\mathcal{H}(\Omega, \alpha) \triangleq\{\overline{\boldsymbol{p}}+\alpha(\boldsymbol{p}-\overline{\boldsymbol{p}}): \boldsymbol{p} \in \Omega\}$. The factor of this deformation, $\alpha$, is called the Similitude Ratio. While expansions are accomplished when $\alpha>1$, contractions result when $0 \leq \alpha<1$. Hereafter, deformations must be interpreted as homothetic expansions or contractions. For purposes of this paper, two uncertainty sets will be called Proportional if there exist a homothetic deformation that relates them, e.g., $\mathcal{R}(\overline{\boldsymbol{p}}, \boldsymbol{m})$ and $\mathcal{R}(\overline{\boldsymbol{p}}, \alpha \boldsymbol{m})$ are proportional sets since $\mathcal{H}(\mathcal{R}(\overline{\boldsymbol{p}}, \boldsymbol{m}), \alpha)=\mathcal{R}(\overline{\boldsymbol{p}}, \alpha \boldsymbol{m})$.

Intuitively, one imagines that a set proportional to the reference set is being deformed until its boundary touches the boundary of the failure domain, i.e., until at least one member of the deformed set is at the verge of violating one or several of the closed-loop requirements (See Figure 
1). Any point where the deforming set touches the failure domain is a Critical Parameter Value (CPV). The CPV, which will be denoted as $\tilde{\boldsymbol{p}}$, might not be unique. The deformed set is called the Maximal Set (MS) and will be denoted as $\mathcal{M}_{\boldsymbol{p}}$. The Critical Similitude Ratio (CSR), denoted as $\tilde{\alpha}$, is the similitude ratio of that deformation. While the CSR is a non-dimensional number, the Parametric Safety Margin (PSM), denoted as $\rho$ and defined in Section IV, is its dimensional equivalent. Both the CSR and the PSM quantify the size of the MS. While the CSR depends on the size of $\Omega$, the PSM does not.

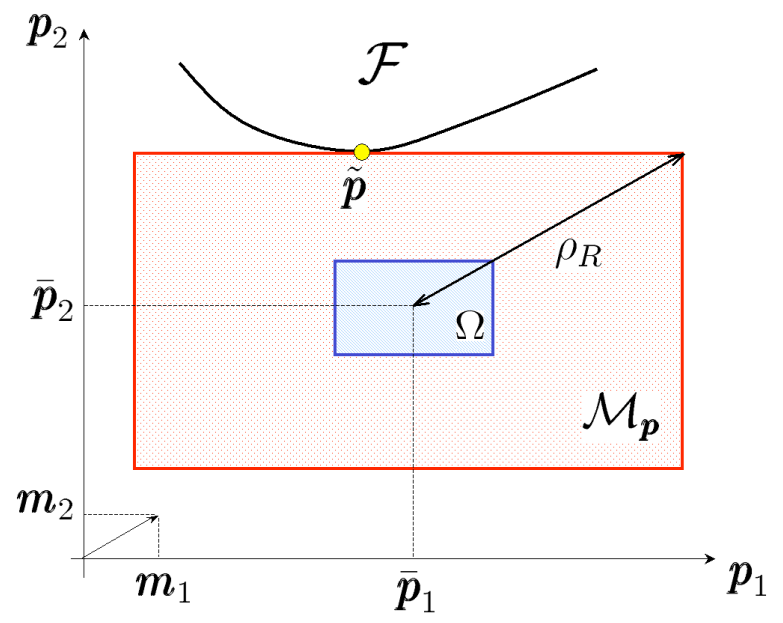

Figure 1. Relevant metrics in the deformation of a rectangular reference set.

The formulations that follow depend on whether the controller with gains $\overline{\boldsymbol{d}}$ satisfies the requirements for the plant at the nominal parameter point (i.e., Nominal Plant) or not. To make this distinction, the following function is introduced

$$
\gamma= \begin{cases}1 & \text { if } \overline{\boldsymbol{d}} \in \mathcal{E} \\ -1 & \text { otherwise }\end{cases}
$$

When $\gamma=-1$, the controller does not satisfy the specifications for the nominal plant. In this case we want to determine the separation between the pair $\langle\overline{\boldsymbol{p}}, \overline{\boldsymbol{d}}\rangle \in \mathcal{F}_{\boldsymbol{p}, \boldsymbol{d}}$ and the non-failure domain $\mathcal{F}_{\boldsymbol{p}, \boldsymbol{d}}^{c}$. The larger the separation the worse the controller. The need for assessing bad controllers results from the desire of searching for optimal ones in an automated fashion. ${ }^{14}$

Formulations that enable the deformation of hyper-spherical and hyper-rectangular sets in the parameter space are presented in the next section. These formulations require solving an optimization problem. Such a problem is non-convex when there are nonlinear parameter dependencies in $\boldsymbol{g}$. In any non-convex optimization problem there is always the possibility of convergence to a local optimum instead of the global optimum. When this occurs, the estimated MS is not fully contained in the non-failure domain and the corresponding figures of merit are larger than those obtained when a global optimum is found. Absolute guarantees are not possible, but a variety of algorithmic safeguards can be used to compensate for this deficiency. For instance, $\boldsymbol{g}$ can be evaluated at few samples points in $\mathcal{M}$, and if some happen to be in the failure domain, they can be used as initial conditions in subsequent searches.

\section{III.A. Deformation of hyper-spheres}

For $\gamma=1$, the CPV for the $j$ th requirement is given by

$$
\tilde{\boldsymbol{p}}^{j}=\underset{\boldsymbol{p}}{\operatorname{argmin}}\left\{\|\boldsymbol{p}-\overline{\boldsymbol{p}}\|: \boldsymbol{g}_{j}(\boldsymbol{p}, \overline{\boldsymbol{d}}) \geq 0\right\},
$$


while the overall CPV is

$$
\tilde{\boldsymbol{p}}=\tilde{\boldsymbol{p}}^{k},
$$

where

$$
k=\underset{1 \leq j \leq \operatorname{dim}(\boldsymbol{g})}{\operatorname{argmin}}\left\{\left\|\tilde{\boldsymbol{p}}^{j}-\overline{\boldsymbol{p}}\right\|\right\} .
$$

Hence, the CPV for each individual requirement is identified, and the closest of these CPVs to the nominal parameter point is the overall CPV.

For $\gamma=-1$, the CPV for the $j$ th requirement is given by

$$
\tilde{\boldsymbol{p}}^{j}=\underset{\boldsymbol{p}}{\operatorname{argmin}}\left\{\|\boldsymbol{p}-\overline{\boldsymbol{p}}\|: \boldsymbol{g}_{j}(\boldsymbol{p}, \overline{\boldsymbol{d}}) \leq 0\right\},
$$

while the overall $\mathrm{CPV}$ is

$$
\tilde{\boldsymbol{p}}=\underset{\boldsymbol{p}}{\operatorname{argmin}}\left\{\|\boldsymbol{p}-\overline{\boldsymbol{p}}\|: \boldsymbol{g}_{j}(\boldsymbol{p}, \overline{\boldsymbol{d}}) \leq 0, j=1, \ldots, \operatorname{dim}(\boldsymbol{g})\right\} .
$$

In this case the overall CPV may not coincide with any of the $\tilde{\boldsymbol{p}}^{j}$ s since this point must be in the intersection of the non-failure domains corresponding to all requirements. If there exists a $j$ such that $\tilde{\boldsymbol{p}}^{j}$ does not exist, meaning that the deformation grew unbounded, the overall CPV does not exist either.

Note that for $\gamma=1$, the nominal point $\overline{\boldsymbol{p}}$ is not in the failure domain, and the CPV $\tilde{\boldsymbol{p}}$ is the parameter point on the boundary of the failure domain that is the closest to $\overline{\boldsymbol{p}}$. Similarly, for $\gamma=-1$ the nominal point $\overline{\boldsymbol{p}}$ is in the failure domain, and the CPV $\tilde{\boldsymbol{p}}$ is the parameter point in the non-failure domain that is the closest to $\overline{\boldsymbol{p}}$. Note that for $\gamma=1$, the CPV is the $\boldsymbol{p}$ value closest to $\overline{\boldsymbol{p}}$ in the failure domain, while for $\gamma=-1$, the CPV is the $\boldsymbol{p}$ value closest to $\overline{\boldsymbol{p}}$ in the nonfailure domain. To prevent the CPV from assuming values leading to infeasible plants, additional constraints must be used in Equation (8) and Equation (11), e.g., if $\boldsymbol{p}_{1}$ is a mass, the constraint $\boldsymbol{p}_{1}>0$ must be imposed. Further note that the critical requirement(s), i.e., any that prevents a larger deformation, is the one corresponding to $\boldsymbol{g}_{k}$.

\section{III.B. Deformation of hyper-rectangles}

Recall that the infinity norm is defined as $\|\boldsymbol{x}\|^{\infty}=\sup _{i}\left\{\left|\boldsymbol{x}_{i}\right|\right\}$. Let us define the $\boldsymbol{m}$-scaled infinity norm as $\|\boldsymbol{x}\|_{\boldsymbol{m}}^{\infty} \triangleq \sup _{i}\left\{\left|\boldsymbol{x}_{i}\right| / \boldsymbol{m}_{i}\right\}$. A distance between the vectors $\boldsymbol{x}$ and $\boldsymbol{y}$ can be defined as $\|\boldsymbol{x}-\boldsymbol{y}\|_{\boldsymbol{m}}^{\infty}$. Using this distance, the hyper-rectangle $\mathcal{R}(\overline{\boldsymbol{p}}, \boldsymbol{m})$ is the unit ball centered at $\overline{\boldsymbol{p}}$, i.e., $\mathcal{R}(\overline{\boldsymbol{p}}, \boldsymbol{m})=\left\{\boldsymbol{p}:\|\boldsymbol{p}-\overline{\boldsymbol{p}}\|_{\boldsymbol{m}}^{\infty} \leq 1\right\}$.

The CPV corresponding to the deformation of the hyper-rectangular set $\Omega=\mathcal{R}(\overline{\boldsymbol{p}}, \boldsymbol{m})$ results from using Equations (8-9) or Equations (11-12) after replacing the Euclidean norm with the $\boldsymbol{m}$ scaled infinity norm. Using the definition of the $\boldsymbol{m}$-scaled infinity norm in Equation (8) leads to

$$
\tilde{\boldsymbol{p}}^{j}=\underset{\boldsymbol{p}}{\operatorname{argmin}}\left\{\max _{1 \leq i \leq \operatorname{dim}(\boldsymbol{p})} \frac{\left|\boldsymbol{p}_{i}-\overline{\boldsymbol{p}}_{i}\right|}{\boldsymbol{m}_{i}}: \boldsymbol{g}_{j}(\boldsymbol{p}, \overline{\boldsymbol{d}}) \geq 0\right\} .
$$

The "max" can be eliminated from the objective function, thereby removing a potential derivative discontinuity, by introducing the similitude ratio $\alpha$ defined earlier

$$
\left\langle\tilde{\boldsymbol{p}}^{j}, \tilde{\alpha}^{j}\right\rangle=\underset{\boldsymbol{p}, \alpha}{\operatorname{argmin}}\left\{\alpha: \boldsymbol{g}_{j}(\boldsymbol{p}, \overline{\boldsymbol{d}})>0, \overline{\boldsymbol{p}}-\alpha \boldsymbol{m} \leq \boldsymbol{p} \leq \overline{\boldsymbol{p}}+\alpha \boldsymbol{m}\right\} .
$$

Hence, for the $\gamma=1$ case, the CPV for each individual constraint function is solved for, and the closest of these CPVs to the nominal parameter point according to the $\boldsymbol{m}$-scaled infinity norm is 
the overall CPV. The statements on the need for additional constraints and on the interpretation of the CPV made in the previous section hold.

Once the CPV has been found, the MS is uniquely determined and its size can be readily measured. Developments for this are presented next.

\section{Figures of Merit for Deterministic Uncertainty Models}

The Spherical PSM is defined as

$$
\rho_{S}(\tilde{\boldsymbol{p}}) \triangleq \gamma\|\tilde{\boldsymbol{p}}-\overline{\boldsymbol{p}}\|,
$$

where $\tilde{\boldsymbol{p}}$ is the CPV resulting from Equations (8) or (9) when $\gamma=1$, or from Equations (11) or (12), when $\gamma=-1$. The corresponding CSR is $\tilde{\alpha}=\left|\rho_{S}\right| / R$, and the MS is given by

$$
\mathcal{M}_{\boldsymbol{p}}=\mathcal{S}(\overline{\boldsymbol{p}}, \tilde{\alpha} R)
$$

If the overall $\mathrm{CPV}$ is used and $\gamma=1, \mathcal{M}_{\boldsymbol{p}}$ is the largest hyper-sphere centered at $\bar{p}$ which fits within the non-failure domain. Conversely, if $\gamma=-1, \mathcal{M}_{\boldsymbol{p}}$ is the the largest hyper-sphere centered on $\overline{\boldsymbol{p}}$ which fits within the failure domain.

On the other hand, the Rectangular PSM is defined as

$$
\rho_{R}(\tilde{\boldsymbol{p}}) \triangleq \gamma \tilde{\alpha}\|\boldsymbol{m}\|
$$

where the CSR is $\tilde{\alpha}=\|\tilde{\boldsymbol{p}}-\overline{\boldsymbol{p}}\|_{\boldsymbol{m}}^{\infty}$ and $\tilde{\boldsymbol{p}}$ is given by the $\boldsymbol{m}$-scaled infinity norm version of Equations (8-9) (i.e. Equation(13)) when $\gamma=1$, or of Equations (11-12) when $\gamma=-1$. The corresponding MS is given by

$$
\mathcal{M}_{\boldsymbol{p}}=\mathcal{R}(\overline{\boldsymbol{p}}, \tilde{\alpha} \boldsymbol{m}) .
$$

As stated previously, if the overall CPV is used and $\gamma=1$, the MS is the largest hyper-rectangle proportional to $\mathcal{R}(\overline{\boldsymbol{p}}, \boldsymbol{m})$ which fits within the non-failure domain $\mathcal{F}^{c}$. Conversely, if $\gamma=-1$, the MS is the largest hyper-rectangle that fits within the failure domain $\mathcal{F}$. If the CPV of the $j$ th requirement is used instead, these remarks apply to $\left(\mathcal{F}^{j}\right)^{c}$ and $\mathcal{F}^{j}$ respectively. For $\gamma=1$, the overall PSM coincides with the smallest individual PSM, i.e., $\rho(\tilde{\boldsymbol{p}})=\min \left\{\rho\left(\tilde{\boldsymbol{p}}^{j}\right)\right\}$. This does not hold when $\gamma=-1$, in which case the smallest individual PSM is just an upper bound to the overall PSM, i.e., $\rho(\tilde{\boldsymbol{p}}) \leq \min \left\{\rho\left(\tilde{\boldsymbol{p}}^{j}\right)\right\}$.

Because the CSR and the PSM measure the size of the MS, their values are proportional to the degree of robustness of the controller associated with $\overline{\boldsymbol{d}}$ to uncertainty in $\boldsymbol{p}$. The CSR is nondimensional, but depends on both the shape and the size of the reference set. The PSM has the same units as the uncertain parameters, and depends on the shape, but not the size, of the reference set. The sign convention enforced by $\gamma$ in Equations $(14,16)$ implies the following. If the PSM takes on a negative value, the controller does not even satisfy the requirements for nominal parameter point. If the PSM is zero, the controller's robustness is practically nil since there are infinitely small perturbations of $\overline{\boldsymbol{p}}$ leading to the violation of at least one of the requirements. If the PSM is positive, the requirements are satisfied for parameter points in the vicinity of the the nominal parameter point. The larger the PSM, the larger the variation from the nominal parameter point to which the uncertain parameter can be subjected without leading to a violation of a requirement. Overall, the larger the PSM the more robust is the controller (i.e., the further the nominal parameter point of the failure domain), with a negative PSM being unacceptable.

Traditionally, robustness analysis is made by setting forth an uncertainty set and checking if the closed-loop performance is satisfactory for all set members. This applies to both, real parameter 
uncertainty and model form-uncertainty (e.g., unmodeled dynamics). A robustness analysis test of this kind based on the developments above is available. ${ }^{12}$

Determination of whether a controller is robust or not for a given hyper-spherical or hyperrectangular set $\Delta$, is a matter of making $\Omega=\Delta$ and calculating the value of the CSR. The controller is robust iff the CSR is greater or equal to one. A controller might turn out to be nonrobust (recall the definition of non-robust) because of an overly-large uncertainty set or because the requirements are too stringent. If the uncertainty model and the design requirements are not relaxed, the only choice is to change the control structure such that a robust controller can be found. This practice however, usually leads to complex, high-order controllers. Other options may be to (i) relax the closed-loop requirements, (ii) reduce the size of the uncertainty set, or (iii) allow for requirement violations with a small probability. Since uncertainty models are never perfect, the closed-loop stability and performance guarantees derived from using a prescribed uncertainty model, e.g., $\Delta$ or $f_{\boldsymbol{p}}(\boldsymbol{p})$, should be interpreted with the same level of rigor used to prescribe such a model.

\section{Figures of Merit for Probabilistic Uncertainty Models}

Metrics and tools for the control verification of systems having a probabilistic uncertainty model are presented next. Note that the only information from the probabilistic model that is used in determining if a controller is robust is the support set of the probabilistic density function and not the actual distribution of the uncertain parameters. Therefore, the developments that follow are suited for controllers that are non-robust. The analysis of such controllers entails quantifying the severity by which the design requirements are violated. In the context of the developments presented thus far, one quantifier for this is the volume of the set $\Delta \cap \mathcal{M}_{p}^{c}$. This metric approximates the failure domain, and evaluates its probability for the case where all parameter realizations are uniformly distributed. Such an assumption is inadequate if one regards parameter realizations closer to the nominal parameter point as more important/likely than the ones further away. To properly account for the relative importance of all parameter realizations, a probabilistic uncertainty model can be used. The value of the joint density function at each particular parameter realization can be interpreted as a measure of our belief that such a realization is the true value of the uncertain parameter. In this setting, a natural quantifier of robustness is the probability of violating the design requirements. This probability, called the Failure Probability, will be denoted as $P[\mathcal{F}]$. The failure probability is zero for robust controllers, while it is greater than zero for non-robust ones. Clearly, the larger $P[\mathcal{F}]$, the more severe the violation. It is worth noticing that small values of $P[\mathcal{F}]$ do not necessarily imply that the design is in the feasible design space, i.e., is it possible to attain $P[\mathcal{F}] \ll 1$ when there exists a $j$ for which $\boldsymbol{g}_{j}(\overline{\boldsymbol{p}}, \boldsymbol{d})>0$.

The main appeal of the developments that follow is that they will enable the comparison of simple controllers along with complex ones from a practical perspective, (e.g., for $99 \%$ of the plants the closed-loop performance of a PID controller may be indistinguishable from the one of a high-order controller). Since no uncertainty model is perfect, one may accept using a non-robust controller with a simple control structure when its corresponding probability of failure is sufficiently small. Forcing the controller to attain zero failure probability may lead to complex controllers whose performance is as good as that of much simpler controllers for most parameter realizations.

As opposed to the contributions in [15-18] on probabilistic controls, the developments herein are not intended to alleviate the computational burden of pursuing worst-case robust stability, but to enable the discrimination among events in the set of all possible closed-loop system performances according to their chance of occurrence. This has the potential to address and alleviate the demands on the controller complexity imposed by worst-case control policies. In this regard, this paper is better aligned with the developments in References [2,19-21] and with the idea of handling some 
of the requirements as soft constraints. ${ }^{22,23}$

Hereafter, we assume that a probabilistic uncertainty model is available. Recall that such a model is prescribed by the joint probability density function $f_{\boldsymbol{p}}(\boldsymbol{p})$, or equivalently by the joint cumulative distribution function $F_{\boldsymbol{p}}(\boldsymbol{p})$, defined over $\Delta$. The framework for the calculation of three probabilistic figures of merit, - the Reliability Index (RI), an upper bound to the failure probability, and the failure probability itself- is presented next.

\section{V.A. Reliability Indices}

The calculation of probabilities can be better performed in spaces other than $\boldsymbol{p}$-space. The most relevant of these spaces is the standard normal space, commonly referred to as the $\boldsymbol{u}$-space. In $\boldsymbol{u}$-space, the multi-variate probability density function becomes an uncorrelated normal density function with zero mean and unit standard deviation, whose value decreases as $\exp \left(-\|\boldsymbol{u}\|^{2} / 2\right)$. For many useful probability distributions, one can find a probability preserving parameter transformation, ${ }^{24}$ denoted hereafter as $\boldsymbol{u}=U(\boldsymbol{p})$, that maps the requirements from $\boldsymbol{p}$-space to $\boldsymbol{u}$-space such that $P[U(\mathcal{F})]=P[\mathcal{F}]$.

The formulations introduced in Section III that enable the deformation of sets in $\boldsymbol{p}$-space can be easily extended to the $\boldsymbol{u}$-space. An obvious parallelism between the concepts, notions and equations introduced earlier and the ones used here is apparent. For instance, if $\Omega=\mathcal{S}(\overline{\boldsymbol{u}}, R)$, and $\gamma=1$, the CPV corresponding to the $j$ th requirement is given by

$$
\tilde{\boldsymbol{u}}^{j}=\underset{\boldsymbol{u}}{\operatorname{argmin}}\left\{\|\boldsymbol{u}-\overline{\boldsymbol{u}}\|: \boldsymbol{g}_{j}\left(U^{-1}(\boldsymbol{u}), \overline{\boldsymbol{d}}\right) \geq 0\right\},
$$

while the overall $\mathrm{CPV}$ is given by

$$
\tilde{\boldsymbol{u}}=\tilde{\boldsymbol{u}}^{k}
$$

where

$$
k=\underset{1 \leq j \leq \operatorname{dim}(\boldsymbol{g})}{\operatorname{argmin}}\left\{\left\|\tilde{\boldsymbol{u}}^{j}-\overline{\boldsymbol{u}}\right\|\right\} .
$$

These equations are analogous to Equations (8-9). A natural choice is $\overline{\boldsymbol{u}}=\mathbf{0}$ because in standard normal space most of the probability is concentrated about the origin (it is mean).

In regard to eliminating infeasible plants when performing deformations in $\boldsymbol{u}$-space, it is sufficient to prescribe a support set $\Delta$ in $\boldsymbol{p}$-space only made of feasible realizations. However, upper bounds on $\|\boldsymbol{u}\|$ should be imposed when searching for the CPV, in order to avoid the divergence and error caused by the numerical evaluation of $U$ and its inverse. These bounds can be chosen large enough so the neglected portion of the $\boldsymbol{u}$-space has negligible probability.

Analogous to the spherical PSM is the Spherical RI, which is given by

$$
\beta_{S}(\tilde{\boldsymbol{u}}) \triangleq \gamma\|\tilde{\boldsymbol{u}}-\overline{\boldsymbol{u}}\|
$$

The corresponding MS is given by

$$
\mathcal{M}_{\boldsymbol{u}}=\mathcal{S}(\overline{\boldsymbol{u}}, \tilde{\alpha} R)
$$

where $\tilde{\alpha}=\left|\beta_{S}\right| / R$. Analogous to the rectangular PSM is the Rectangular RI, which is given by

$$
\beta_{R}(\tilde{\boldsymbol{u}}) \triangleq \gamma \tilde{\alpha}\|\boldsymbol{m}\|
$$

where $\tilde{\alpha}=\|\tilde{\boldsymbol{u}}-\overline{\boldsymbol{u}}\|_{\boldsymbol{m}}^{\infty}$. The corresponding MS is given by

$$
\mathcal{M}_{\boldsymbol{u}}=\mathcal{R}(\overline{\boldsymbol{u}}, \tilde{\alpha} \boldsymbol{m})
$$


If the controller is robust, none of the optimization problems posed in this section has a finite solution since the failure domain in $\boldsymbol{u}$-space is empty. The RI exists if and only if the failure probability is non-zero, i.e., the controller is non-robust. As before, for $\gamma=1$ the overall RI coincides with the smallest individual RI, e.g., $\beta(\tilde{\boldsymbol{u}})=\min \left\{\beta\left(\tilde{\boldsymbol{u}}^{j}\right)\right\}$, while for $\gamma=-1$ the smallest individual RI is an upper bound to the overall RI, e.g., $\beta(\tilde{\boldsymbol{u}}) \leq \min \left\{\beta\left(\tilde{\boldsymbol{u}}^{j}\right)\right\}$. Note that if the overall CPV is used and $\gamma=1, \mathcal{M}_{\boldsymbol{u}}$ is the largest set proportional to $\Omega$ which fits within $U\left(\mathcal{F}^{c}\right)$. Conversely, if $\gamma=-1, \mathcal{M}_{\boldsymbol{u}}$ is the largest set proportional to $\Omega$ that fits within $U(\mathcal{F})$. If the CPV of the $j$ th requirement is used instead, these remarks apply to $U\left(\left(\mathcal{F}^{j}\right)^{c}\right)$, and $U\left(\mathcal{F}^{j}\right)$ respectively.

The developments of this paper require setting both an uncertainty model and a reference set. Guidance on how to select the latter is given next. For $\boldsymbol{p}$-deformations, the relative levels of uncertainty among the components of $\boldsymbol{p}$ are fully prescribed by the $\boldsymbol{p}$-space reference set which, for this part of the discussion, will be denoted as $\Omega_{\boldsymbol{p}}$. In contrast, for $\boldsymbol{u}$-deformations these levels depend on both, the $\boldsymbol{u}$-space reference set $\Omega_{\boldsymbol{u}}$, and the probability density function $f_{\boldsymbol{p}}(\boldsymbol{p})$. The manner in which the geometry of $\Omega_{\boldsymbol{u}}$, and the shape of $f_{\boldsymbol{p}}(\boldsymbol{p})$ determines these levels is exemplified in Figures 2 and 3. Therein, unimodal and bimodal density functions with $\Omega_{\boldsymbol{u}}=\mathcal{S}(\mathbf{0}, R)$, and $\Omega_{\boldsymbol{u}}=\mathcal{R}(\mathbf{0}, \mathbf{1})$ are considered. The subplots in the left column show the probability density function. A homothetic deformation in $\boldsymbol{u}$-space can be seen as a deformation in $\boldsymbol{p}$-space where the boundary of an expanded set is the image under $U^{-1}$ of the points on the surface of $\mathcal{M}_{\boldsymbol{u}}$ that attain a fixed RI value. The center and right columns of subplots show these boundaries, to be referred to as RI contours, for various values of the spherical and rectangular RI.

Note that (i) images under $U^{-1}$ of proportional sets in $\boldsymbol{u}$-space do not correspond to proportional sets in $\boldsymbol{p}$-space, (ii) the RI contours do not coincide with those of $f_{\boldsymbol{p}}(\boldsymbol{p})$, (iii) the center and the aspect ratio of the rectangular RI contours change with the value of the RI, and (iv) $U^{-1}\left(\mathcal{M}_{\boldsymbol{u}}\right)$ approaches $\Delta$ as the RI approaches infinity. It is apparent that the geometry of $\Omega_{\boldsymbol{u}}$ affects the relative levels of uncertainty. To preserve the levels set by $f_{\boldsymbol{p}}(\boldsymbol{p}), \Omega_{\boldsymbol{u}}$ must be a hyper-sphere or a hyper-cube centered at the origin. For instance, if $\boldsymbol{p}$ is a normally distributed random vector having the mean $\overline{\boldsymbol{p}}$, and covariance matrix $\operatorname{diag}\left\{\boldsymbol{\sigma}^{2}\right\}, \mathcal{R}(\overline{\boldsymbol{p}}, \alpha \boldsymbol{\sigma})=U^{-1}(\mathcal{R}(\mathbf{0}, \alpha \mathbf{1}))$. Therefore, the selection of $\Omega_{\boldsymbol{u}}=\mathcal{R}(\mathbf{0}, \mathbf{1})$ enables that $\boldsymbol{\sigma}$, variable that describes the relative levels of uncertainty in $f_{\boldsymbol{p}}(\boldsymbol{p})$, be also the aspect ratio $\boldsymbol{m}$ of the rectangular RI contours. Note however, that the geometry of the reference set can be adjusted as desired. The developments in the next sections indicate that reference sets leading to larger maximal sets are desirable. Such sets may not preserve the uncertainty levels prescribed by $f_{\boldsymbol{p}}(\boldsymbol{p})$.

Strategies for the calculation of upper bounds to the failure probability based on the deformations of sets in $\boldsymbol{p}$ - and $\boldsymbol{u}$-space are presented next.

\section{V.B. Upper Bounds to Failure Probability}

In this section we use the MS calculated above to derive analytical bounds to the failure probability. The following Lemma provides the basic mathematical background for this.

Lemma 1 (Bounding Interval). Let $\psi \triangleq 1-P[\mathcal{M}]$, where $\mathcal{M}$ is the $M S$. The bounding interval $\mathcal{I}$, which satisfies $P[\mathcal{F}] \in \mathcal{I}$, is given by $\mathcal{I}=(0, \psi]$ for $\gamma=1$, and by $\mathcal{I}=[1-\psi, 1)$ otherwise.

Proof: Assume that $\gamma=1$. Since $P[\mathcal{F}]+P\left[\mathcal{F}^{c}\right]=1$, and $\mathcal{M} \subset \mathcal{F}^{c}$ so $P[\mathcal{M}] \leq P\left[\mathcal{F}^{c}\right]$. It follows that $P[\mathcal{F}] \leq 1-P[\mathcal{M}]=\psi$. Since for $\gamma=-1, \mathcal{M}$ is chosen so that $\mathcal{M} \subset \mathcal{F}$, it follows that $P[\mathcal{F}] \geq 1-\psi$.

Because these bounds result from calculating the probability of an event, and in contrast to several other bounds available in the literature, their value always lies between zero and one. Note that there is a bounding interval for each reference set. Denote the left and right end points of the 

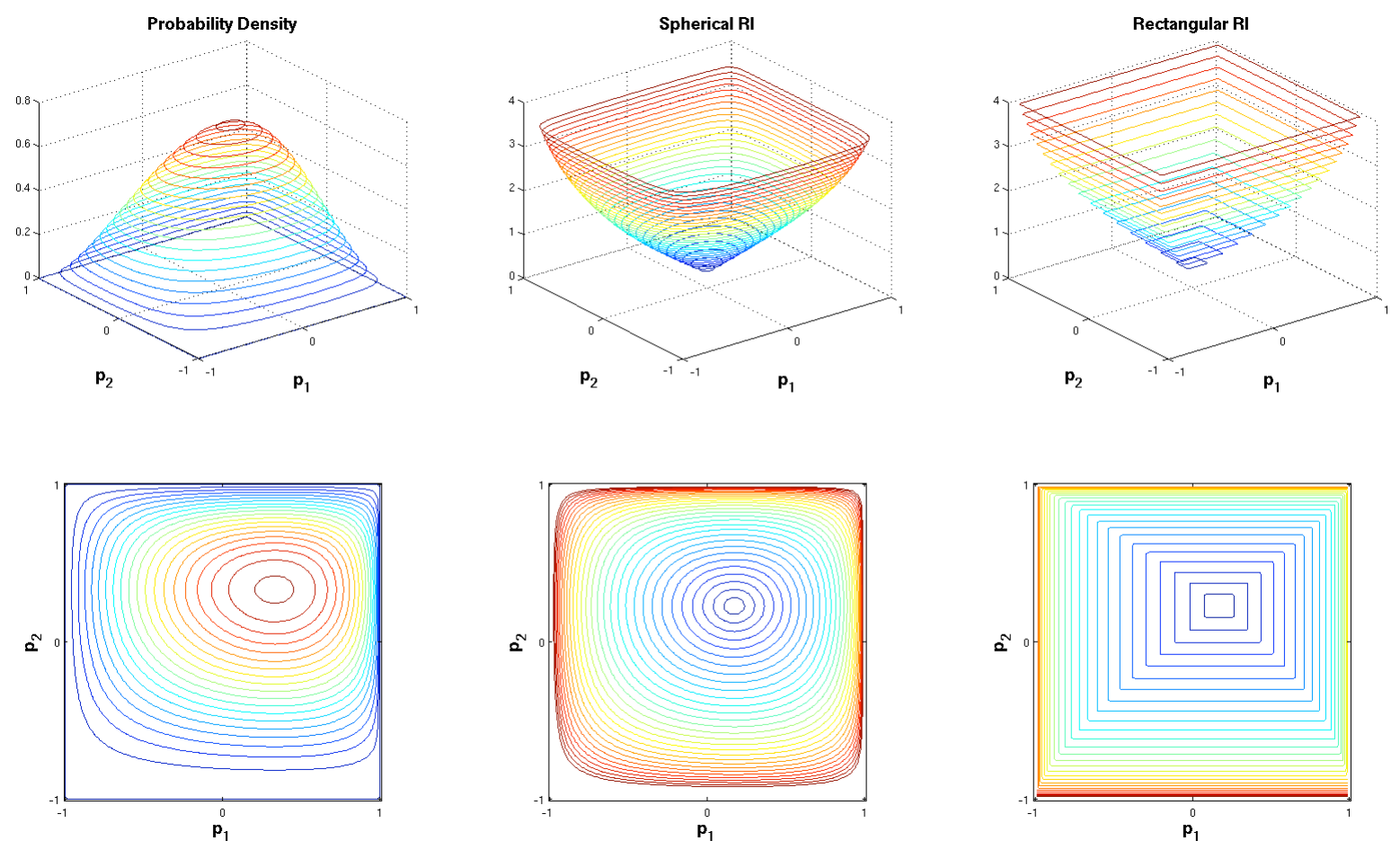

Figure 2. RI contours for a unimodal probability density function.

bounding interval by by $\mathcal{I}_{l}$ and $\mathcal{I}_{r}$ respectively. If $\mathcal{I}$ denotes the bounding interval corresponding to all requirements and $\mathcal{I}^{j}$ the one corresponding to the $j$ th requirement, $\mathcal{I}=\left(0, \min \left\{\mathcal{I}_{r}^{j}\right\}\right]$ for $\gamma=1$, and $\mathcal{I}=\left[\mathcal{I}_{l}, 1\right)$, where $\mathcal{I}_{l} \leq \max \left\{\mathcal{I}_{l}^{j}\right\}$, for $\gamma=-1$. Hereafter, our discussion will concentrate on $\gamma=1$, in which case $\psi$ is an upper bound to $P[\mathcal{F}]$.

When the deformation leading to the MS takes place in $\boldsymbol{p}$-space, we will use the notation

$$
\psi_{\boldsymbol{p}}=1-P\left[\mathcal{M}_{\boldsymbol{p}}\right]
$$

If the components of $\boldsymbol{p}$ are independent random variables and $\Omega$ is hyper-rectangular, $P\left[\mathcal{M}_{\boldsymbol{p}}\right]$ can be evaluated analytically in a straightforward manner.

Lemma 2 (Hyper-rectangles in $\boldsymbol{p}$-space). Let $\rho_{R}$ be the rectangular PSM corresponding to the deformation of the reference set $\Omega=\mathcal{R}(\overline{\boldsymbol{p}}, \boldsymbol{m})$, and to the constraint set $\boldsymbol{g}(\boldsymbol{p}, \overline{\boldsymbol{d}}) \geq \mathbf{0}$. Denote the Cumulative Distribution Function of the probabilistic uncertainty model $F_{\boldsymbol{p}}$, where $\boldsymbol{p}$ is a vector of independent random variables. In this context, Equation (24) leads to

$$
\psi_{\boldsymbol{p}}\left(\overline{\boldsymbol{p}}, \boldsymbol{m}, \rho_{R}\right)=1-\prod_{i=1}^{\operatorname{dim}(\boldsymbol{p})} F_{\boldsymbol{p}_{i}}\left(\overline{\boldsymbol{p}}_{i}+\frac{\rho_{R} \boldsymbol{m}_{i}}{\|\boldsymbol{m}\|}\right)-F_{\boldsymbol{p}_{i}}\left(\overline{\boldsymbol{p}}_{i}-\frac{\rho_{R} \boldsymbol{m}_{i}}{\|\boldsymbol{m}\|}\right) .
$$

The proof of this Lemma is trivial, and results from evaluating the probability of $\mathcal{M}_{p}^{c}$ for $\mathcal{M}_{\boldsymbol{p}}=\mathcal{R}(\overline{\boldsymbol{p}}, \tilde{\alpha} \boldsymbol{m})$. The main advantage of using Equation (25) is that the MS, and therefore the PSM, are independent of the probabilistic uncertainty model assumed. This implies that bounds corresponding to arbitrary uncertainty models are trivial to evaluate since they will only require changing $F_{\boldsymbol{p}}$ in Equation (25). 

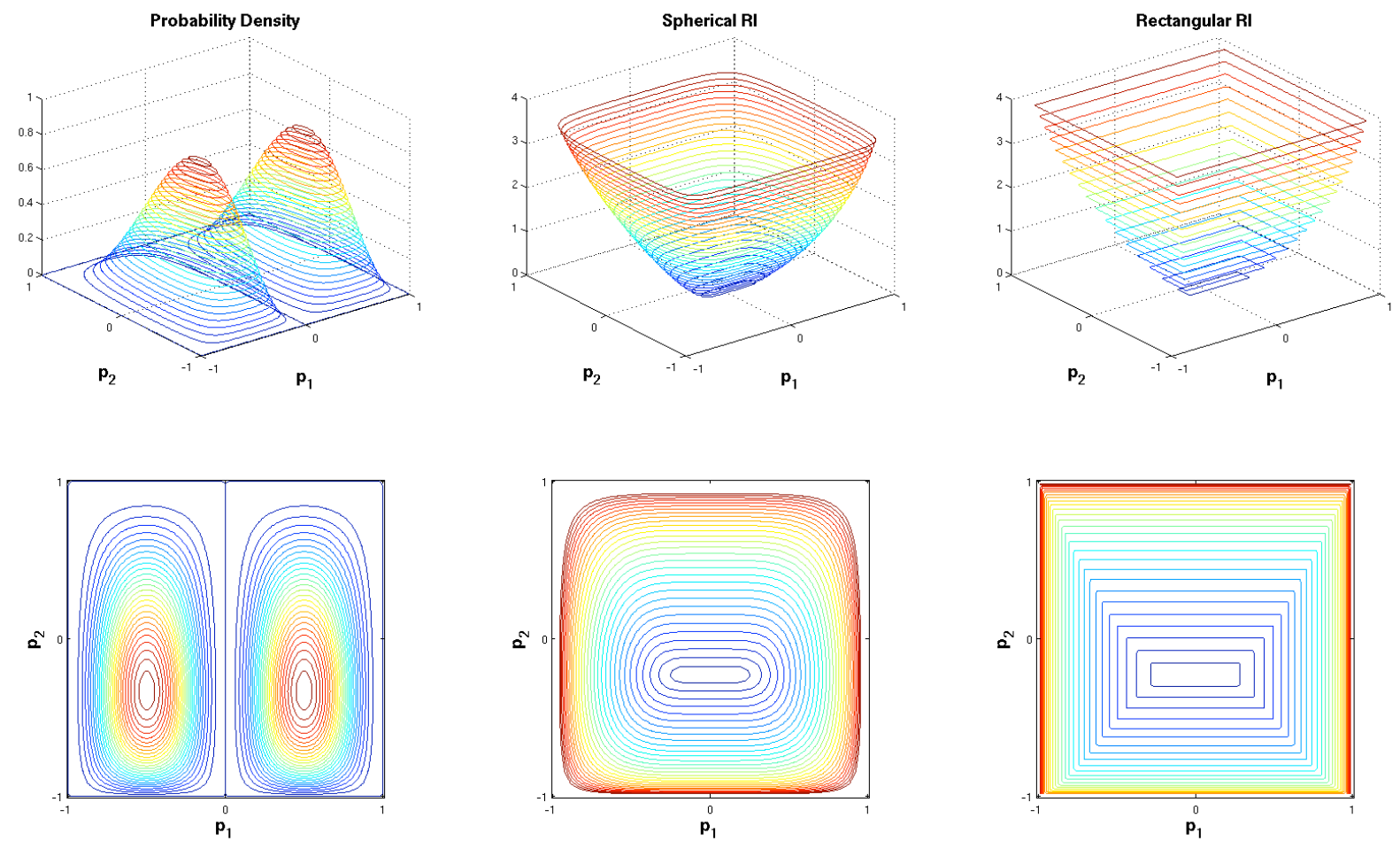

Figure 3. RI contours for a bimodal probability density function.

Tighter upper bounds are obtained if the reference set chosen leads to a larger $P[\mathcal{M}]$. Experience has shown that this can be more easily attained by working in the standard normal space. In particular, deformations of reference sets in $\boldsymbol{u}$-space leading to maximal sets containing the vicinity of the origin usually produce smaller bounds. For these deformations we will use the notation

$$
\psi_{\boldsymbol{u}}=1-P\left[\mathcal{M}_{\boldsymbol{u}}\right] .
$$

While $\psi_{\boldsymbol{p}}$ can be estimated analytically for arbitrary hyper-rectangular sets, $\psi_{\boldsymbol{u}}$ can also be estimated for hyper-spherical sets centered at $\overline{\boldsymbol{u}}=\mathbf{0}$. The following two Theorems, whose proofs are given in Reference [13], present the corresponding closed-form expressions.

Theorem 1 (hyper-spheres in $\boldsymbol{u}$-space). Let $\beta_{S}$ be the spherical RI corresponding to the deformation of the reference set $\Omega=\mathcal{S}(\mathbf{0}, R)$, and the constraint set $\boldsymbol{g}\left(U^{-1}(\boldsymbol{u}), \overline{\boldsymbol{d}}\right) \geq \mathbf{0}$. Let $l=\operatorname{dim}(\boldsymbol{p})$. In this case, Equation (26) is equivalent to

$$
\psi_{\boldsymbol{u}}\left(\beta_{S}\right)=1-\Lambda_{l}\left(\left|\beta_{S}\right|\right),
$$

where $\Lambda_{l}(r)$, which is the cumulative distribution function of a chi-distributed random variable with $l$ degrees of freedom, is given by

$$
\Lambda_{l}(r)= \begin{cases}\operatorname{erf}\left(\frac{r}{\sqrt{2}}\right)-\sqrt{\frac{2}{\pi}}\left(\frac{r^{l-2}}{(l-2) ! !}+\frac{r^{l-4}}{(l-4) ! !}+\cdots+\frac{r}{1 ! !}\right) e^{-r^{2} / 2} & \text { if } r \geq 0, l \text { odd } \\ 1-\left(\frac{r^{l-2}}{(l-2) ! !}+\frac{r^{l-4}}{(l-4) ! !}+\cdots+\frac{r^{2}}{2 ! !}+1\right) e^{-r^{2} / 2} & \text { if } r \geq 0, l \text { even } \\ 0 & \text { otherwise }\end{cases}
$$


and $n$ !! is the double factorial ${ }^{\mathrm{d}}$.

Theorem 2 (hyper-rectangles in $\boldsymbol{u}$-space). Let $\beta_{R}$ be the rectangular $R I$ corresponding to the deformation of the reference set $\Omega=\mathcal{R}(\overline{\boldsymbol{u}}, \boldsymbol{m})$, and the constraint set $\boldsymbol{g}\left(U^{-1}(\boldsymbol{u}), \overline{\boldsymbol{d}}, t\right) \geq \mathbf{0}$. In this case, Equation (26) is equivalent to

$$
\psi_{\boldsymbol{u}}\left(\overline{\boldsymbol{u}}, \boldsymbol{m}, \beta_{R}\right)=1-\prod_{i=1}^{\operatorname{dim}(\boldsymbol{p})} \Phi\left(\overline{\boldsymbol{u}}_{i}+\frac{\left|\beta_{R}\right| \boldsymbol{m}_{i}}{\|\boldsymbol{m}\|}\right)-\Phi\left(\overline{\boldsymbol{u}}_{i}-\frac{\left|\beta_{R}\right| \boldsymbol{m}_{i}}{\|\boldsymbol{m}\|}\right),
$$

where $\Phi$ is the cumulative distribution function of the univariate standard normal random variable.

Recall that $\psi_{\boldsymbol{p}} \in(0,1]$ and $\psi_{\boldsymbol{u}} \in(0,1]$. For a given $f_{\boldsymbol{p}}(\boldsymbol{p}), \boldsymbol{g}, \overline{\boldsymbol{d}}$, we have no general rule that determines which reference set leads to the smallest upper bound. However, we know that the maximal sets leading to small upper bounds contain points where $\|\boldsymbol{u}\|$ is small. For a different uncertainty model, the bounds in Equation (26) require solving another optimization problem in order to identify a new $\beta_{R}$. This is in sharp contrast to the bounds in Equation (25) where the MS is independent of the uncertainty model. Note that while Lemma 2 can be applied to robustand non-robust controllers, Theorems 1 and 2 are only applicable to non-robust ones. This is so because for robust controllers $\mathcal{M}_{\boldsymbol{u}}$ is unbounded. Equations (25), (27) and (28) lead to guaranteed upper bounds to the failure probability.

\section{V.C. Failure Probability}

The problem of finding $P[\mathcal{F}]$, or equivalently $P[U(\mathcal{F})]$, is usually difficult since it requires evaluating a multi-dimensional integral over a complex integration domain. Methods for estimating the failure probability include sampling based techniques, such as Monte Carlo sampling, the First Order Reliability Method, ${ }^{24}$ and the hybrid method. ${ }^{13}$

The hybrid method, which is used in the example of Section VI, approximates the failure probability by combining sampling with the upper bounds introduced above. Once the MS has been determined, an arbitrary sample point can be easily tested for membership in this set. If the sample point lies within the MS, it is unnecessary to go through the computational expense of evaluating $\boldsymbol{g}$, since the outcome is now known. The numerical advantage of using this method is a consequence of not having to evaluate $\boldsymbol{g}$ at these realizations. The method is especially suitable when the number of evaluations of $\boldsymbol{g}$ required to determine the MS is considerably less than the number of samples falling within the MS. The efficiency of the hybrid method increases with the absolute value of the RI. In addition, the accuracy of the method using $n$ samples outside the MS is comparable to the one of Monte Carlo using $N=$ floor $(n / \psi)$ samples. In the context of control analysis, a particularly attractive feature of the hybrid method is that its efficiency and accuracy does not depend on the robustness of the controller. ${ }^{25}$ This sharply contrasts with the case in Monte Carlo-based methods, where accurate robustness assessments (i.e., those based on statistics having small confidence intervals) demand a number of simulations that grow exponentially with the robustness of the controller. In other words, the better the robustness, the smaller the $P[\mathcal{F}]$ and the larger the number of samples required to estimate this probability accurately. Details on the implementation of the hybrid method and its analysis are available in References $[13,25]$.

Now that all the figures of merit have been introduced, it is worth noticing a conceptual difference in the way they measure robustness. While the PSMs, the RIs, and the upper bounds to the

\footnotetext{
${ }^{\mathrm{d}}$ Recall that the double factorial is defined as

$$
n ! != \begin{cases}n \cdot(n-2) \cdots 5 \cdot 3 \cdot 1 & n>0 \text { and odd } \\ n \cdot(n-2) \cdots 6 \cdot 4 \cdot 2 & n \geq 0 \text { and even } \\ 1 & n=-1,0\end{cases}
$$
}


failure probability have a notion of robustness based on the closeness of the nominal parameter point to the failure domain, the failure probability itself quantifies the portion of all possible realizations falling into the failure domain. As such, the estimation of $P[\mathcal{F}]$ does not require the definition of a nominal parameter point or a nominal plant, and designs attaining small failure probabilities may have their corresponding failure domain very close to the realizations where $f_{\boldsymbol{p}}(\boldsymbol{p})$ is the largest. Designs that attain both small $P[\mathcal{F}]$ and large separation between the nominal parameter point and the failure domain are best suited for the hybrid method because of its usage of the failure bounding sets. Notice that this dual notion of robustness cannot be assessed or pursued by using Monte Carlo sampling.

\section{Example: Benchmark Robust Control Problem}

\section{VI.A. Problem Statement}

The verification and comparative analysis of controllers designed for the robust control challenge problem posed in the 1990 American Control Conference [26] are considered here. The benchmark system, shown in Figure 4, is a two-mass/spring system with a non-collocated sensor actuator pair. Several design problems were posed based on this setting. In all of them, stability and performance

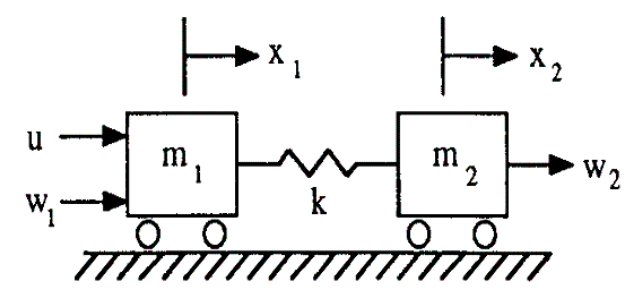

Figure 4. Two-mass spring system.

requirements in the time domain were prescribed for plants with uncertain masses and stiffness whose values lie within a bounded set. As in Reference [27], additional sources of uncertainty are considered herein to fully exercise the scope of the methodology. We added a non-linear spring with constant $k_{n}$, a time delay $\tau$ denoting a first order lag between controller command and actuator response, and a multiplicative loop-gain uncertainty $f$ resulting from variation in sensors, control gain, and/or actuator failure. The state space plant model is

$$
\begin{aligned}
\dot{x}_{1} & =x_{3} \\
\dot{x}_{2} & =x_{4} \\
\dot{x}_{3} & =\frac{k}{m_{1}}\left(x_{2}-x_{1}\right)+\frac{k_{n}}{m_{1}}\left(x_{2}-x_{1}\right)^{3}+\frac{f u}{m_{1}}, \\
\dot{x}_{4} & =\frac{k}{m_{2}}\left(x_{1}-x_{2}\right)+\frac{k_{n}}{m_{2}}\left(x_{1}-x_{2}\right)^{3}+\frac{w_{2}}{m_{2}}, \\
\tau \dot{u} & =u_{c}-u .
\end{aligned}
$$

While the output $\mathbf{z}$ and the observed variable $\mathbf{y}$ are both equal to $x_{2}$, only the disturbance $w_{2}$ will be active. The uncertain parameter vector is $\boldsymbol{p}=\left[m_{1}, m_{2}, k, k_{n}, \tau, f\right]^{T}$ whose nominal value is $\overline{\boldsymbol{p}}=[1,1,1,0,0,1]^{T}$. Note that the nominal values of the additional parameters lead to the plant used in the original benchmark problem. In order to prevent deformations leading to infeasible plants, the constraints $m_{1}>0, m_{2}>0, k>0, \tau>0$ and $f>0$ are imposed on the optimization problem used to calculate the CPVs. The specifications imposed on the closed-loop system are 
1. Local closed-loop stability.

2. Settling time: the response to a unit-impulse must fall between \pm 0.1 after $15 \mathrm{~s}$.

3. Control saturation: the control signal corresponding to the impulse response must fall between \pm 1 .

In the context of this paper, the corresponding set of constraints is

$$
\boldsymbol{g}=\left[\max _{1 \leq l \leq n_{p}}\left\{\Re\left(s^{i}\right)\right\}, \quad \max _{t>15}\{|z(t)|\}-0.1, \quad \max _{t>0}\{|u(t)|\}-1\right]^{T},
$$

where $s^{i}$ is a closed-loop pole of the linearized system and $\Re(\cdot)$ is the real part operator. Eleven controllers were designed for the above problem by several authors. The controllers have been designed using several different methods, including robust $H_{\infty}$, loop-transfer recovery, imaginaryaxis shifting, constrained optimization, structured covariance, game theory, the internal model principle $^{27,28}$ and $\mu$-synthesis. ${ }^{29}$ A Monte Carlo-based analysis of some of these controllers is available in Reference [27].

The state space representation of a controller is given by

$$
\begin{aligned}
\dot{\mathbf{x}}_{c} & =\mathbf{A}_{c} \mathbf{x}_{c}+\mathbf{B}_{c} \mathbf{y}, \\
u_{c} & =\mathbf{C}_{c} \mathbf{x}_{c}+\mathbf{D}_{c} \mathbf{y},
\end{aligned}
$$

where $\mathbf{x}_{c}$ is the controller state, $u_{c}$ is the actuator command, and $\mathbf{A}_{c}, \mathbf{B}_{c}, \mathbf{C}_{c}$, and $\mathbf{D}_{c}$ are the controller matrices. The controllers considered here are the ones labeled as $A, B, C, D, E, F$, and $H$ in Reference [27], and the controllers designed for problems one and two in Reference [28] and Reference [29]. In this paper, the controllers from Reference [28] will be labeled as $W_{1}$ and $W_{2}$, and those from Reference [29] will be labeled as $B_{1}$ and $B_{2}$.

\section{VI.B. Control Verification}

In this section we perform the control verification of the above controllers. In the context of this example, the CPV is the critical combination of uncertain physical parameters, nonlinearity, time delay, and control effectiveness degradation for which the closed-loop specifications are at the verge of being violated (providing they are satisfied for the nominal plant).

The deformation of the reference set $\mathcal{R}(\overline{\boldsymbol{p}}, \boldsymbol{m})$ is considered first. The relative levels of uncertainty among the parameters are assumed to be $\boldsymbol{m}=[4,4,5,2,1,2]^{T}$, e.g., we expect four times more uncertainty in the value of the masses than in the value of the time delay. Note that even though the values in $\boldsymbol{m}$ are subjective, the resulting MS is always fully contained in the non-failure domain. Table 1 presents the stability margins and the PSMs corresponding to the three requirements. According to the results, only $W_{1}, B_{1}$ and $B_{2}$ satisfy the requirements for the nominal plant. While $W_{2}$ has the best figure of merit in regard to stability and control saturation, the settling time requirement is best satisfied by controller $D$. The gain and phase margins for the nominal plant are not reliable indicators of robust stability because they are independent of the way in which the plant depends on $\boldsymbol{p}$. Note that they are not always proportional to the PSM. The controller attaining the best compromise is $B_{2}$, design for which $\rho_{R}=0.038$.

Assessments using a probabilistic uncertainty model are presented next. For this, we assume that $m_{1}, m_{2}, k, k_{n}, \tau$ and $f$ are independent, Beta-distributed random variables with shape parameters, $[5,5],[5,5],[2,3.7],[6,6],[0.3,5]$, and $[0.5,1.5]$, having the support sets $[0,2],[0,2],[0.5,2]$, $[-0.5,0.5],[0,0.1]$ and $[0.5,1.5]$, respectively. This probabilistic model has been set such that $\overline{\boldsymbol{p}}$ and $U^{-1}(\mathbf{0})$ are in $\mathcal{E}$. The ranges of variation of the parameters and the shapes of the distributions are assigned according to engineering judgment. 
Table 1. Stability margins, and rectangular PSMs.

\begin{tabular}{|c|c|c|c|c|c|}
\hline Controller & $\begin{array}{c}\text { Gain } \\
{[\mathrm{db}]}\end{array}$ & $\begin{array}{c}\text { Phase } \\
{[\mathrm{deg}]}\end{array}$ & $\begin{array}{c}\text { Stability } \\
\rho_{R}\left(\tilde{\boldsymbol{p}}^{1}\right)\end{array}$ & $\begin{array}{c}\text { Settling time } \\
\rho_{R}\left(\tilde{\boldsymbol{p}}^{2}\right)\end{array}$ & $\begin{array}{c}\text { Control } \\
\rho_{R}\left(\tilde{\boldsymbol{p}}^{3}\right)\end{array}$ \\
\hline$A$ & 2.5 & -20.6 & 0.320 & -0.019 & 0.368 \\
\hline$B$ & 3.2 & 26.7 & 0.490 & -0.175 & 0.523 \\
\hline$C$ & 3.3 & 26.6 & 0.501 & -0.189 & 0.533 \\
\hline$D$ & 15.1 & 57.2 & 0.562 & 0.212 & $-\infty$ \\
\hline$E$ & 2.4 & 22.2 & 0.394 & -0.259 & 0.233 \\
\hline$F$ & 5.2 & 23.9 & 0.419 & 0.012 & -3.277 \\
\hline$H$ & 3.4 & 24.8 & 0.510 & -0.005 & 0.524 \\
\hline$W_{1}$ & 3.3 & 24.5 & 0.501 & 0.001 & 0.517 \\
\hline$W_{2}$ & 6.0 & 34.2 & 0.754 & -0.024 & 0.639 \\
\hline$B_{1}$ & 1.8 & 18.9 & 0.300 & 0.020 & 0.003 \\
\hline$B_{2}$ & 2.8 & 27.2 & 0.437 & 0.038 & 0.141 \\
\hline
\end{tabular}

Deformations of hyper-spherical reference sets in $\boldsymbol{u}$-space lead to the results in Table 2. According to the RIs, the controller $D$ is the one with best stability and settling time characteristics while $W_{2}$ has the best figure of merit for control saturation. As in the previous case, only $W_{1}$, $B_{1}$ and $B_{2}$ satisfy the requirements for the nominal plant while $B_{2}$ is the most robust of all controllers. Conclusions based in the RI coincide with the ones based on the upper bound, so results corresponding to the latter have been omitted.

The values of $P\left[\mathcal{F}^{j}\right]$, calculated using the hybrid method with $n=1000$ samples, are shown in Table 3. Recall that $P[\mathcal{F}]$ does not depend on whether the nominal point is in the feasible design space or not. According to this metric, $D$ is the best controller in stability and settling time, while $W_{2}$ is the best for control saturation. Values of $P[\mathcal{F}]$ for $W_{1}, B_{1}$ and $B_{2}$, which were the only controllers satisfying the requirements for the nominal plant, were calculated using $n=2000$ samples. The resulting values, which are $0.908,0.980$ and 0.873 respectively, indicate that $B_{2}$ is the controller with best robustness characteristics. Since the failure probabilities are large, the advantage of using the hybrid method, as compared to sampling is moderate.

The control assessments above are consistent even though they use different uncertainty models and figures of merit. These analyses determine not only which is the limiting design requirement for each controller, but more importantly, how the levels of robustness corresponding to all requirements compare among themselves.

\section{Concluding Remarks}

Figures of merit for control verification that quantify a controller's ability to satisfy the closedloop requirements in the presence of uncertain parameters are proposed. The framework developed 
Table 2. Spherical RIs.

\begin{tabular}{|c|c|c|c|}
\hline Controller & $\begin{array}{c}\text { Stability } \\
\beta_{S}\left(\tilde{\boldsymbol{u}}^{1}\right)\end{array}$ & $\begin{array}{c}\text { Settling time } \\
\beta_{S}\left(\tilde{\boldsymbol{u}}^{2}\right)\end{array}$ & $\begin{array}{c}\text { Control } \\
\beta_{S}\left(\tilde{\boldsymbol{u}}^{3}\right)\end{array}$ \\
\hline$A$ & 0.665 & -0.037 & 0.913 \\
\hline$B$ & 0.992 & -0.319 & 1.169 \\
\hline$C$ & 1.01 & -0.336 & 1.191 \\
\hline$D$ & 2.366 & 0.598 & $-\infty$ \\
\hline$E$ & 0.690 & -3.517 & 0.374 \\
\hline$F$ & 1.627 & 0.025 & $-\infty$ \\
\hline$H$ & 1.050 & -0.009 & 1.174 \\
\hline$W_{1}$ & 1.027 & 0.0009 & 1.152 \\
\hline$W_{2}$ & 2.147 & -0.072 & 2.287 \\
\hline$B_{1}$ & 0.497 & 0.030 & 0.005 \\
\hline$B_{2}$ & 0.852 & 0.066 & 0.236 \\
\hline
\end{tabular}

is applicable to systems whose functional dependence on the uncertain parameters is arbitrary, and for which stability and performance requirements are present. The figures of merit are the parametric safety margin, the reliability index, the failure probability, and upper bounds to this probability. The tools used to calculate these metrics are based on the solution to optimization problems whose formulation and solution does not introduce conservatism. The parametric safety margin enables quantification of robustness when uncertainty is prescribed as a set. The other metrics, for which a probabilistic uncertainty model is required, account for the chance of occurrence of any given plant within the uncertainty set and therefore for the likelihood of unsatisfactory closed-loop performance. As compared to sampling-based methods, the efficiency and accuracy of the tools proposed is substantially better. This improvement is particularly advantageous when the controllers are highly robust, a case when the computational expense of an accurate sampling-based analysis is impractical. This framework enables a fair comparison of controllers designed using different methods and assumptions. The justification of adopting complex control architectures and the determination of the consequences of violating such assumptions are aspects that can be naturally evaluated using this framework.

\section{Acknowledgment}

This work was supported by the Integrated Resilient Aircraft Control Project of NASA. 
Table 3. Failure Probabilities via the hybrid method.

\begin{tabular}{|c|c|c|c|}
\hline Controller & $\begin{array}{c}\text { Stability } \\
P\left[\mathcal{F}^{1}\right]\end{array}$ & $\begin{array}{c}\text { Settling time } \\
P\left[\mathcal{F}^{2}\right]\end{array}$ & $\begin{array}{c}\text { Control } \\
P\left[\mathcal{F}^{3}\right]\end{array}$ \\
\hline$A$ & 0.272 & 0.971 & 0.158 \\
\hline$B$ & 0.143 & 0.957 & 0.119 \\
\hline$C$ & 0.130 & 0.967 & 0.104 \\
\hline$D$ & 0.007 & 0.333 & 1 \\
\hline$E$ & 0.219 & 0.999 & 0.426 \\
\hline$F$ & 0.080 & 0.871 & 1 \\
\hline$H$ & 0.138 & 0.914 & 0.188 \\
\hline$W_{1}$ & 0.119 & 0.907 & 0.174 \\
\hline$W_{2}$ & 0.014 & 0.875 & 0.010 \\
\hline$B_{1}$ & 0.313 & 0.976 & 0.601 \\
\hline$B_{2}$ & 0.196 & 0.798 & 0.462 \\
\hline
\end{tabular}




\section{References}

${ }^{1}$ Bryson, A. E. and A.Mills, R., "Linear-Quadratic-Gaussian Controllers with Specified Parameter Robustness," AIAA Journal of Guidance, Control and Dynamics, Vol. 21, No. 1, 1998, pp. 11-18.

${ }^{2}$ Tenne, D. and Singh, T., "Efficient Minimax Control Design for Prescribed Parameter Uncertainty," AIAA Journal of Guidance, Control and Dynamics, Vol. 27, No. 6, 2004, pp. 1009-1016.

${ }^{3}$ Skogestad, S. and Postlethwaite, I., Multivariable feedback control, John Wiley and Sons, Chichester, England, 1996.

${ }^{4}$ Darligton, J., Pantelides, C., Rustem, B., and Tanyi, B., "An algorithm for constrained nonlinear optimization under uncertainty," Automatica, Vol. 35, 1999, pp. 217-228.

${ }^{5}$ Rustem, B. and Nguyen, Q., "An algorithm for the inequality constrained minimax problem," SIAM Journal of Optimization, Vol. 8, No. 1, 1998, pp. 265-283.

${ }^{6}$ deGaston, R. and Sofonov, M., "Exact calculation of the multiloop stability margin," IEEE Transactions on Automatic Control, Vol. 33, No. 2, 1988, pp. 156-171.

${ }^{7}$ Sideris, A. and Pena, R., "Robustness Margin Calculation with Dynamic and Real Parametric Uncertainties," Proceedings of the American Control Conference, 1988, pp. 1201-1206.

${ }^{8}$ Barmish, B. R., "New Tools for Robustness Analysis," Proceedings of the 27th Conference on Decision and Control, Austin, Texas, 1988, pp. 1-6.

${ }^{9}$ Boyd, S. L., Ghaoui, L. E., Feron, E., and Balakrishnan, V., Linear matrix inequalities in systems and control theory, SIAM, Philadelphia, PA, 1994.

${ }^{10}$ Barmish, B. R. and Shcherbakov, P. S., "A dilation method for robustness problems with nonlinear parameter dependence," Proceedings of the American Control Conference, Denver, Colorado, 2003, pp. 3834-3839.

${ }^{11}$ Babayigit, A., Ross, B. R., and Shcherbakov, P. S., "On robust stability with nonlinear parameter dependence: some benchmark problems illustrating the dilation integral method," Proceedings of the 2004 American Control Conference, Boston, Massachusetts, 2004, pp. 2671-2673.

${ }^{12}$ Crespo, L. G., Giesy, D. P., and Kenny, S. P., "Robust Analysis and Robust Design of Uncertain Systems," AIAA Journal, Vol. 46, No. 2, 2008.

${ }^{13}$ Crespo, L. G., Giesy, D. P., and Kenny, S. P., "Reliability-based Analysis and Design via Failure Domain Bounding," Structural Safety, 2008, Accepted for publication pending final revision.

${ }^{14}$ Crespo, L. G., Kenny, S. P., and Giesy, D. P., "A Verification-driven approach to Control Analysis and Tunning," AIAA Guidance Navigation and Control Conference, Honolulu, Hawaii, USA, August 18-21 2008, p. TBD.

${ }^{15}$ Blondel, V. and J.N.Tsitsiklis, "A survey of computational complexity results in systems and control," Automatica, Vol. 36, 2000, pp. 1249-1274.

${ }^{16}$ Calafiore, G., Dabbene, F., and Tempo, R., "Randomized Algorithms for Probabilistic Robustness with Real and Complex Structured Uncertainty," IEEE Transactions on Automatic Control, Vol. 45, No. 12, December 2000, pp. 2218-2235.

${ }^{17}$ Polyak, B. and Tempo, R., "Probabilistic robust design with linear quadratic regulators," Systems and Control Letters, Vol. 43, 2001, pp. 343-353.

${ }^{18}$ Raghavan, V. and Barmish, B. R., "Stability of Systems with Random Parameters," Proceedings of the 45th Conference on Decision and Control, San Diego, California, 2006, pp. 3180-3185.

${ }^{19}$ Wang, Q. and Stengel, R. F., "Robust control of nonlinear systems with parametric uncertainty," Automatica, Vol. 38, 2002, pp. 1591-1599.

${ }^{20}$ Crespo, L. G. and Kenny, S. P., "Reliability-based control design for uncertain systems," AIAA Journal of Guidance, Control, and Dynamics, Vol. 28, No. 4, 2005.

${ }^{21}$ Wang, Q. and Stengel, R. F., "Robust Nonlinear Flight Control of a High Performance Aircraft," IEEE Transactions on Automatic Control, Vol. 13, No. 1, 2005, pp. 15-26.

${ }^{22}$ Lagoa, C. M., "A convex-parameterization of risk-adjusted stabilizing controllers," Automatica, Vol. 39, 2003, pp. 1829-1835.

${ }^{23}$ Barmish, B. R. and Shcherbakov, P., "On avoiding vertexization of robustness problems: the approximate feasibility concept," IEEE Transactions on Automatic Control, Vol. 47, No. 5, 2002, pp. 819-824.

${ }^{24}$ Rackwitz, R., "Reliability analysis, a review and some perspectives," Structural Safety, Vol. 23, 2001, pp. 365395.

${ }^{25}$ Giesy, D. P., Crespo, L. G., and Kenny, S. P., "Approximation of Failure Probability using Conditional Sampling," 12th AIAA/ISSMO Multidisciplinary Analysis and Optimization Conference, Victoria, Canada, 10-12 September 2008, p. TBD.

${ }^{26}$ Wie, B. and Bernstein, D., "A Benchmark Problem for Robust Control Design," Proceedings of the 1990 American Control Conference, Vol. 1, San Diego, CA, USA, 1990, pp. 961-962.

19 of 20 
${ }^{27}$ Stengel, R. F. and Morrison, C., "Robustness of Solutions to a Benchmark Control Problem," AIAA Journal of Guidance, Control and Dynamics, Vol. 15, No. 5, 1992, pp. 1060-1067.

${ }^{28}$ Wie, B., Liu, Q., and Byun, K.-W., "Robust H-infinity control synthesis method and its application to Benchmark problems," AIAA Journal of Guidance, Control, and Dynamics, Vol. 15, No. 5, 1992, pp. 1140-1148.

${ }^{29}$ Braatz, R. and Morari, M., "Robust Control for a Noncollocated Spring-Mass System," Journal of Guidance, Control and Dynamics, Vol. 15, No. 5, 1992, pp. 1103-110. 